\title{
When a Goitre is a Thyroid Lymphoma
}

\author{
Mariana Gonçalves, Elsa Gaspar, Lèlita Santos, Armando Carvalho \\ Internal Medicine Department, Centro Hospitalar e Universitário de Coimbra, Portugal
}

Received: $18 / 11 / 2018$

Accepted: $24 / 11 / 2018$

Published: $21 / 12 / 2018$

How to cite this article: Gonçalves M, Gaspar E, Santos L, Carvalho A. When a goitre is a thyroid lymphoma. EJCRIM 2018;5: doi:10.12890/2018_000999.

Conflicts of Interests: The Authors declare that there are no competing interests.

This article is licensed under a Commons Attribution Non-Commercial 4.0 License

\section{ABSTRACT}

Primary thyroid lymphoma is a rare cause of both thyroid malignancy and extra-nodal lymphoma. It typically presents as a rapidly enlarging goitre with compressive symptoms. Histological analysis of a biopsy specimen is necessary to confirm the diagnosis. Awareness of this disease is important for early diagnosis and appropriate treatment. A 55-year-old man was admitted due to a 3-month history of cervical enlargement, facial and periorbital oedema and dyspnoea, apparently due to a superior vena cava syndrome. However, cervical ultrasound showed asymmetrical thyroid enlargement, lobulated contours and a hypoechogenic texture. A neck CT scan showed calibre reduction of the superior vena cava. The diagnosis of primary thyroid lymphoma was confirmed by open biopsy of the thyroid gland. The patient started treatment with rituximab with clinical improvement.

\section{LEARNING POINTS}

- Primary thyroid lymphoma is rare, accounting for less than $5 \%$ of thyroid malignancies and less than $2 \%$ of extra-nodal lymphomas, but should be suspected in the presence of a rapidly enlarging goitre.

- Fine needle aspiration biopsy is the initial technique of choice, but core needle biopsy or open surgical biopsy may be necessary to establish the diagnosis.

- Treatment and prognosis are dependent on accurate histological classification, with the disease generally treated successfully with chemotherapy.

\section{KEYWORDS}

Cervical enlargement, primary thyroid lymphoma, extra-nodal lymphoma

\section{INTRODUCTION}

Primary thyroid lymphoma (PTL) is an uncommon tumour, estimated to account for approximately $5 \%$ of all thyroid neoplasms. Diffused large B-cell lymphoma and extra-nodal marginal zone lymphoma of mucosa-associated lymphoid tissue constitute more than $90 \%$ of cases ${ }^{[1,2]}$. Follicular lymphoma (FL) accounts for 3-5\% of all PTLs, and is one of the most common forms of non-Hodgkin lymphoma (NHL), with an estimated incidence in Europe of 2.18 cases per 100,000 persons per year. Thyroid NHL accounts for approximately $1.2-1.7 \%$ of all $\mathrm{NHL}{ }^{[3,4]}$. Patients with PTL most commonly present in their sixties, with women being more frequently affected (female:male ratio ranging from 2:1 to 8:1). It typically presents as a rapidly enlarging goitre with compressive symptoms and cervical lymphadenopathy. A definitive diagnosis of PTL requires histological tissue analysis ${ }^{[4,5]}$. It is very curable without the need for extensive surgery, so early recognition and correct treatment is vital[ ${ }^{[3-5]}$. 


\section{CASE PRESENTATION}

We describe the case of a 55-year-old man admitted with a progressive increase in cervical diameter, associated with facial and periorbital oedema and dyspnoea that had started 3 months previously. He reported several episodes of syncope on standing or sitting up rapidly, on elevation of his arms above his head and during bathing. He did not have fever, weight loss, anorexia, asthenia or sweating. He had a personal history of hypertension, dyslipidaemia and multinodular goitre. Chronic medications included levothyroxine (0.1 mg/day), irbesartan (150 $\mathrm{mg} /$ day), atorvastatin (10 mg/day) and pantoprazole (40 mg/day). There was no history of alcohol abuse or illicit drug use. He did not refer high-risk sexual exposures and did not report recent travel. Regarding family history, his brother had been diagnosed with a lymphoma in middle age and had died.

On physical evaluation, the patient was afebrile and haemodynamically stable, with cervical, facial, periorbital and upper-extremity oedema, an enlarged thyroid, and venous dilation of the neck and chest wall, suggesting a superior vena cava syndrome. Blood work-up only revealed a slightly increased C-reactive protein of $2.31 \mathrm{mg} / \mathrm{dl}$ (normal value $<0.5 \mathrm{mg} / \mathrm{dl}$ ), and the thyroid hormones were within the normal range.

The patient underwent a cervical ultrasound that revealed an asymmetrical thyroid, with greatly enlarged dimensions, discreetly lobulated contours and a markedly hypoechogenic texture. Multiple ganglionic formations were observed with bilaterally oval morphology, some of them necrotic, the largest on the left being $15 \times 15 \mathrm{~mm}$ in size and on the right $17 \times 11 \mathrm{~mm}$, with the morphological and dimensional criteria of adenopathies. A neck CT scan showed densification of fat in the upper mediastinum, calibre reduction of the superior vena cava, two adenopathies measuring $22 \times 14 \mathrm{~mm}$ and $17 \times 12 \mathrm{~mm}$ in a pre-tracheal location, and also fat densification in the plane of the carina (Fig. 1). Consequently, phlebography of the central vessels was performed and showed pre-occlusive stenosis of the superior vena cava involving the brachiocephalic venous trunks, with left brachiocephalic venous trunk thrombosis. Two stents were placed with recovery of permeability and evident clinical improvement. Fine needle aspiration cytology of cervical adenopathy was inconclusive. Thus, surgical intervention was performed with open biopsy of the thyroid gland, whose histological result was compatible with follicular thyroid B lymphoma.

For staging the disease, a fluorodeoxyglucose (FDG) positron emission tomography (PET) scan was performed, revealing high-grade hypermetabolic lymphomatous disease, with supra-diaphragmatic lymph node involvement forming adenopathic conglomerates without cleavage planes with the thyroid gland (Fig. 2). The patient was treated with rituximab with disease remission.
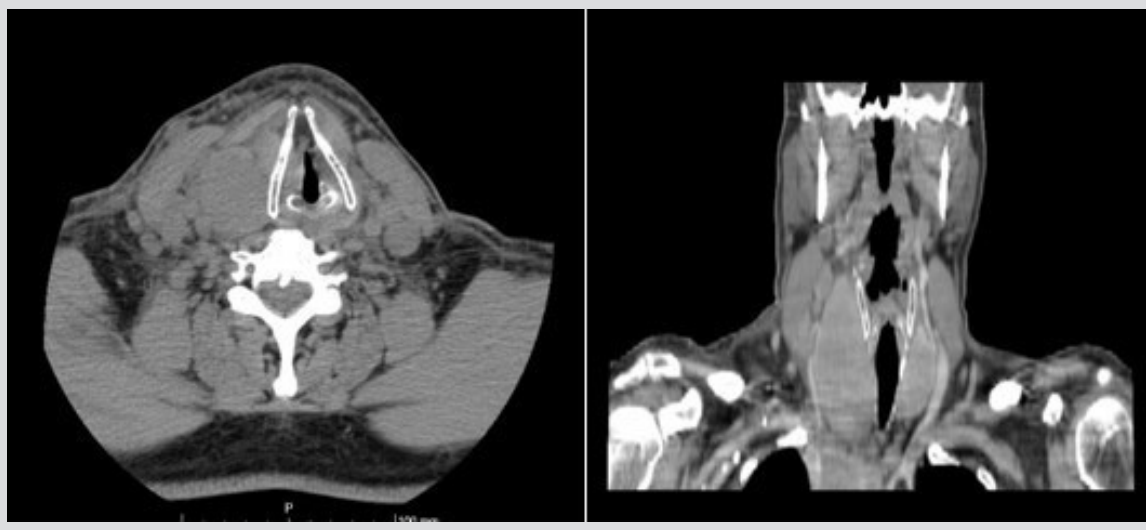

Figure 1. Neck CT scan showing densification of fat in the upper mediastinum, calibre reduction of the superior vena cava and fat densification in the plane of the carina

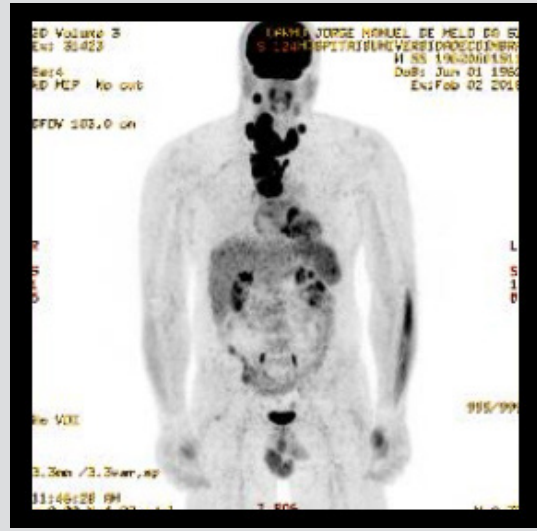

Figure 2. PET showing high-grade hypermetabolic lymphomatous disease, with supra-diaphragmatic lymph node involvement forming adenopathic conglomerates without cleavage planes with the thyroid 


\section{DISCUSSION}

PTL is a rare type of thyroid cancer, accounting for 1-2\% of all extra- nodal lymphomas, with FL representing only 3-5\% of all PTLs. There are several different types of lymphoma and determining the exact type is essential for treatment selection and prognosis ${ }^{[1,3,4]}$.

The most common presentation is a rapidly enlarging thyroid mass. Other symptoms such as dyspnoea, dysphagia and hoarseness may arise as a result of mass effects. Rarely stridor or superior vena cava obstruction may occur. B-type symptoms such as weight loss and night sweats occur less commonly and have been reported in approximately $20 \%$ of patients ${ }^{[1,4]}$.

Despite its rarity, PTL should always be considered in a patient with a rapidly growing goitre or thyroid nodules, bearing in mind that about $30-60 \%$ of patients are biochemically euthyroid at presentation ${ }^{[3-5]}$.

Cervical ultrasonography is a standard initial imaging modality, showing thyroid patterns that may suggests the diagnosis. Once the diagnosis of PTL has been established, a CT scan of the entire body is necessary in order to stage the patient accurately. FDG PET scanning appears to be a good imaging modality for assessing the extent of $\mathrm{PTL}[3,4]$.

Histological analysis is necessary to confirm the diagnosis, with the best method being to perform a tissue biopsy. Fine needle aspiration biopsy is the initial technique of choice but is often not able to obtain enough cells for an accurate diagnosis. In these cases, core needle biopsy or open surgical biopsy may be necessary ${ }^{[1,3]}$.

The optimal treatment for PTL remains controversial because of limited evidence. As with other lymphomas, PTL is sensitive to both chemotherapy and radiotherapy. Most PTL is treated successfully with chemotherapy with rituximab alone or in combination with other chemotherapeutic agents. Therefore, surgery is rarely required for treatment. The prognosis is generally excellent but is dependent on the subtype of lymphoma $a^{[1,3,4]}$.

Although PTL is a rare cause of both thyroid malignancy and extra-nodal lymphoma, awareness of this disease is important in order to achieve an early diagnosis and implement suitable treatment ${ }^{[4]}$.

\section{REFERENCES}

1. Rosai J, DeLellis RA. Carcangiu ML. AFIP atlas of tumor pathology: tumors of the thyroid and parathyroid glands. Rockville, MD: ARP Press; 2014

2. Derringer GA, Thompson LD, Frommelt RA. Malignant lymphoma of the thyroid gland: a clinicopathologic study of 108 cases. Am J Surg Pathol 2000:24:623-639.

3. Stein SA, Wartofsky L. Primary thyroid lymphoma: a clinical review. J Clin Endocrinol Metab 2013;98:3131-3138.

4. Walsh S, Lowery AJ, Evoy D, McDermott EW, Prichard RS. Thyroid lymphoma: recent advances in diagnosis and optimal management strategies. Oncologist 2013;18:994-1003.

5. Nam M, Shin JH, Han BK. Thyroid lymphoma: correlation of radiologic and pathologic features. J Ultrasound Med 2012;31:589-594. 